\title{
Parámetros Genéticos e Índices de Selección para Corderosy Borregas Hampshire bajo Crianza Intensiva en un Rebaño de la Costa Central del Perú
}

\author{
Genetic Parameters and Selection Indices for Hampshire Lambs and Ewes under \\ an Intensive Breeding in a Flock of the Central Coast of Peru
}

\author{
Iván Carrera ${ }^{1,3}$, Juan Chávez ${ }^{1,4}$, Elmer Meza ${ }^{2}$
}

\section{Resumen}

\begin{abstract}
El presente estudio tuvo por objetivo caracterizar con fines de mejoramiento genético un rebaño ovino de la raza Hampshire ubicado en la costa central del Perú. Se estimaron parámetros genéticos y fenotípicos para las características Tamaño de Camada al Nacimiento (TCN), Peso de la Camada al Destete (PCD), Peso al Destete a los 2 Meses (PD), Peso de Beneficio a los 5 Meses (PB) y Ganancia de Peso del Destete a los 5 Meses (GPD5), y se elaboraron índices de selección para corderos $\left(\mathrm{I}_{1}\right.$ e $\left.\mathrm{I}_{2}\right)$ y borregas $\left(\mathrm{I}_{3}\right)$ utilizando datos acumulados de 2002 a 2004. Se estimaron componentes de variancia y covarianza mediante la metodología de Máxima Verosimilitud Restringida (REML), bajo un modelo mixto que incluyó como factores fijos el sexo, tipo de parto, estación y año de nacimiento, y como efecto aleatorio el padre. Los valores medios fenotípicos para peso corporal en corderos y los productivos y reproductivos en borregas se ubicaron en el estándar racial para sistemas de crianza con nivel tecnológico medio. Los parámetros genéticos correspondieron a los señalados en la literatura, salvo las heredabilidades de $\operatorname{TCN}(0.26 \pm 0.28)$ y $\mathrm{PB}(0.36 \pm 0.21)$, que fueron superiores e inferiores, respectivamente, a valores previamente reportados. Las correlaciones genéticas y fenotípicas entre pesos mostraron valores positivos medios a altos. Los índices de selección para corderos tuvieron la expresión: $\mathrm{I} 1=4.782 * \mathrm{~PB}+2.864 * \mathrm{GPD} 5$ e I2 $=8.263 * \mathrm{PD}+3.182 * \mathrm{~PB}+4.164 * \mathrm{GPD} 5$, con coeficientes de correlación de 0.415 y 0 . 527. respectivamente; y, para las borregas: $\mathrm{I} 3=\overline{\mathrm{X}_{r}}$ $+[\mathrm{n} * 0.0153 / 1+(\mathrm{n}-1) * 0.0153] *\left(\overline{\mathrm{X}_{i}}-\overline{\mathrm{X}_{*}}\right)$, los cuales podrían ser aplicados con fines de selección pero con un relativo grado de eficiencia.
\end{abstract}

Palabras clave: Hampshire; corderos; borregas; parámetros genéticos; selección; índices

\footnotetext{
${ }^{1}$ Departamento de Producción Animal, Facultad de Zootecnia, Universidad Nacional Agraria La Molina, Lima, Perú

${ }^{2}$ Municipalidad Provincial de Cangallo, Ayacucho, Perú. E-mail: elzar2112@hotmail.com

${ }^{3}$ E-mail: ivancarrerasegouin@yahoo.es

${ }^{4}$ E-mail.jchavez@lamolina.edu.pe
} 
The study aimed to characterize for genetic improvement purposes an ovine Hampshire flock located in the central coast of Peru. Genetic and phenotypic parameters were estimated for Birth Litter Size (TCN), Litter Weaning Weight (PCD), 2-Month Weaning Weight (PD), 5-Month Slaughter Weight (PB), and Body Weight Gain from Weaning to 5 Months Old (GPD5); and selection indices for lambs $\left(\mathrm{I}_{1}\right.$ and $\left.\mathrm{I}_{2}\right)$ and ewes $\left(\mathrm{I}_{3}\right)$ were developed using data collected between 2002 and 2004. Components of the variance and covariance were estimated using the Restricted Maximum Likelihood method (REML), under a mixed model that included sex, type of birth, season and year of birth as fixed effects, and sire as random effect. Phenotypic mean values estimated for body weight traits in lambs and productive and reproductive traits in ewes were similar to the breed standard reared under medium technology systems. The estimated genetic parameters corresponded to those reported in the literature except the $\operatorname{TCN}(0.26 \pm 0.28)$ and $\mathrm{PB}(0.36 \pm 0.21)$ heritabilities which were above and below the values commonly reported elsewhere, respectively. All genetic and phenotypic correlations among body weights showed medium to high positive values. Lamb selection indices had the expressions: $\mathrm{I} 1=4.782 * \mathrm{~PB}+2.864 * \mathrm{GPD} 5$ e I2 $=$ $8.263 * \mathrm{PD}+3.182 * \mathrm{~PB}+4.164 * \mathrm{GPD} 5$, with correlation coefficients of 0.415 and 0.527 , respectively; and for ewes $\mathrm{I} 3=+\left[\mathrm{n} * 0.0153 / 1+(\mathrm{n}-1)^{*} 0.0153\right] *\left(\overline{\mathrm{X}_{i}}-\overline{\mathrm{X}_{r}}\right)$, which could be applied for selection purposes but with relative efficiency.

Key words: Hampshire; lambs; ewes; genetic parameters; selection; index

\section{INTRODUCCIÓN}

En la actualidad, el precio de la lana ha mejorado como consecuencia de una menor oferta debido a la reducción de la población mundial de ovinos; sin embargo, la tendencia de incrementar la producción de carne ovina de buena calidad se mantiene por una mayor demanda de productos cárnicos, derivada de la mejora de los ingresos de la población mundial, especialmente en la clase media y media alta (Salgado, 2012; García, 2013). Esta tendencia también se aprecia en el Perú, dada la reducción del $23 \%$ de la población de ovinos entre los censos agropecuarios de 1994 y 2012, teniéndose este último año una existencia de 9523198 cabezas (INEI, 2013).

El ovino Hampshire pertenece al grupo denominado «caras negras», que tiene su origen en Inglaterra, siendo muy populares por sus características cárnicas (Fraser y Stamp, 1989). Dentro de este grupo sobresale la raza Hampshire Down, la cual en su formación tuvo influencia de la raza Southdown, también inglesa, caracterizándose por buena velocidad de crecimiento y conversión alimenticia (OSU, 2014).

El Hampshire es un típico animal productor de carne, de cuerpo ancho, profundo y compacto, costillas bien arqueadas, dorso y lomo rectos, anchos y bien cubiertos de carne, grupa horizontal y amplia, cola gruesa, extremidades cortas, aplomos correctos ( $\mathrm{Sa}-$ les, 1998; citado por Castillo, 2003). Los carneros pesan entre 100 y $135 \mathrm{~kg}$ y las ovejas de 70 a $90 \mathrm{~kg}$. Producen de 3 a $3.5 \mathrm{~kg}$ de lana de mediana calidad, pudiendo presentar fibras negras en el vellón (Aliaga, 2006).

En los Estados Unidos, corderos Hampshire bajo crianza semiextensiva, comparados con corderos de otras seis razas de ovinos, entre las que se encontraron razas de carne como la Suffolk y la Dorset, lograron pesos vivos ligeramente mayores al promedio a los seis meses de edad $(56.6 \mathrm{~kg}$ respecto a $55.9 \mathrm{~kg}$ ); pero sus rendimientos en 
carcasa $(53.5 \%)$, conformación (7.54 puntos) y acabado (6.92 puntos) fueron superiores a todas ellas, mostrando una ventaja comparativa para la producción de carne de calidad (Dickerson, 1972). Asimismo, en crianza intensiva en la costa peruana se han obtenido corderos para beneficio a temprana edad, reportándose ganancias diarias de hasta 480 g (Aliaga, 2006; Castillo, 2003). Complementariamente, se ha observado que las borregas son muy dóciles, prolíficas, y poseen buena producción de leche y habilidad materna (Aliaga, 2006).

En la producción de carne de ovino se reconocen dos fases: i) la maternal, en la que son relevantes las características de fertilidad, prolificidad, producción de leche y habilidad materna; y ii) la del corderaje, en la cual interesan la velocidad de crecimiento, el peso corporal, la conformación y el acabado (Mueller, 2001). La calidad de la carne depende, entre otros aspectos, de la cantidad, naturaleza y distribución de grasa y músculo, pudiendo ser controlada eficazmente con la edad al beneficio (Mueller, 1996). Todas estas características responden al cruzamiento expresando diversos grados de respuesta, la que ha sido dimensionada por Bianchi et al. (2001) en el Uruguay.

Los parámetros genéticos son claves para describir y caracterizar poblaciones, permitiendo la aplicación de estrategias de selección, incidiendo tanto en los valores genotípicos como genéticos -valor de cría o habilidad transmisora - de los animales, y poder mejorar las características de interés (Falconer, 1981). Dependen tanto de la población como de las circunstancias ambientales en las que se desarrolla, considerándose entre estos a la heredabilidad y la repetibilidad para las características individuales, y las correlaciones fenotípicas y genéticas para pares de ellas (Cardellino y Rovira, 1987).

Las correlaciones predicen la dirección y la tasa del cambio de características, distintas a las que están bajo selección, lo cual es crucial para decidir sobre cuáles de ellas incluir cuando, por estrategia de mejoramiento genético, se opta por desarrollar y aplicar un índice de selección (James, 1987). No se han reportado estimaciones de parámetros genéticos en el país para el Hampshire; sin embargo, hay reportes para el Suffolk en Estados Unidos (Notter, 1998), raza muy cercana al Hampshire (Crispim et al., 2013).

Grupos de productores nacionales iniciaron la producción de carne ovina de manera intensiva con razas especializadas. Se comenzó a registrar información de los animales y del manejo, así como los egresos e ingresos que demandaba la crianza y su funcionamiento, teniendo como uno de sus objetivos el elaborar planes de selección con miras a elevar el nivel genético productivo de sus animales. Con base en esto, el presente trabajo tuvo por objetivo estimar parámetros genéticos de características consideradas relevantes para la producción de carne ovina de calidad, aplicables en la elaboración de índices para la selección de corderos y borregas Hampshire en sistemas de crianza intensiva en la costa central del Perú, orientados a la mejora de su eficiencia productiva. Este trabajo puede ser considerado el primer estudio exploratorio en el país de la caracterización genética de un rebaño de ovinos de la raza Hampshire orientado a la producción de carne de calidad en condiciones intensivas, bajo alimentación de forraje y concentrado.

\section{Materiales y Métodos}

\section{Localización y Animales}

El estudio se realizó en el establecimiento ganadero Agropecuaria Arequipa S.A., ubicado en el distrito de Lurín, provincia y departamento de Lima, a una altitud de 185 msnm. Los rangos de temperatura en el lugar fluctúan entre 14 y $27^{\circ} \mathrm{C}$, con una humedad relativa promedio del $70 \%$. La crianza es en forma estabulada, bajo un programa de manejo propio de sistemas intensivos. La ali- 
Cuadro 1. Número de registros usados en la estimación de las medias fenotípicas y parámetros genéticos

\begin{tabular}{lc}
\hline Procedencia & $\begin{array}{c}\text { Registros } \\
\text { (n) }\end{array}$ \\
\hline Padres & \\
Total padres & 7 \\
Total madres & 110 \\
Progenie (sexo) & \\
Machos & 52 \\
Hembras & 90 \\
Progenie (tipo de parto) & \\
Simples & 76 \\
Dobles & 66 \\
Progenie (año) & \\
2002 & 12 \\
2003 & 51 \\
2004 & 79 \\
Progenie (estación) & \\
Primavera & 48 \\
Verano & 34 \\
Otoño & 11 \\
Invierno & 49 \\
\hline
\end{tabular}

mentación fue a base de concentrados elaborados con insumos locales y forrajes como maíz-chala.

El plantel estuvo compuesto por carneros Puros de Pedigrí (PDP) y Puros por Cruza (PPC), que representaron el 53.3 y $46.7 \%$, respectivamente, donde la diferencia radica en si estaban inscritos en los Registros Genealógicos Zootécnicos del Perú (R.G.Z.P), mas no en su potencial genético. El $46 \%$ fue adquirido en Puno y Arequipa, el 7\% en la Universidad Nacional Agraria La Molina (UNALM) y el $47 \%$ restante fue generado en la propia granja, dando prioridad a los descendientes de padres PDP. En el caso de las hembras, el $80 \%$ fue PPC, pues a diferencia de los machos, la granja no tiene como priori- dad su inscripción en los R.G.Z.P. Asimismo, el 95\% de ellas fueron generadas en la granja y el $5 \%$ restante fue adquirido en las ferias ganaderas del interior del país.

\section{Origen y Estructura de los Datos}

Se utilizó información proveniente de los registros de manejo y producción del rebaño de 2002 a 2004. Se obtuvieron 228 registros pero finalmente quedaron 142 al descartarse varios de ellos por: i) valores inconsistentes en magnitud, dependiendo del carácter medido; ii) animales con información incompleta desde el nacimiento hasta el beneficio; iii) reducido número de hijos por padre (menor de cinco); y iv) escasas observaciones de un carácter, en particular por parte del padre, entre otros. Se logró estandarizar a una misma cantidad de padres en cada uno de los tres años evaluados, haciendo el seguimiento a sus descendientes. Finalmente, la información depurada fue estructurada considerando efectos ambientales sistemáticos como año, estación, sexo, tipo de parto, padre y madre (Cuadro 1).

\section{Caracterización Genética y Reproductiva}

Se utilizó información de los libros de antecedentes de compra de animales, certificados de registros genealógicos, así como tarjetas de identificación y productividad, que permitió conocer: i) los promedios fenotípicos de los caracteres productivos en machos y hembras; ii) la estructura y organización del plantel reproductor; y iii) el esquema de mejoramiento y de difusión del material genético superior.

Además, se contó con datos de los libros de registro de manejo y producción, que permitieron estimar los índices productivos y reproductivos de las borregas (prolificidad, número de partos, corderos nacidos y logrados anualmente por madre, intervalo entre partos, edad al primer servicio y al parto, natalidad bruta y mortalidad). 


\section{Estimación de parámetros genéticos}

Se estimó la heredabilidad y error estándar para: Tamaño de Camada al Nacimiento (TCN), Peso de la Camada al Destete (PCD), Peso al Destete a los 2 meses (PD), Peso al Beneficio (PB), y Ganancia de Peso del Destete a los 5 meses (GPD5); así como las correlaciones genéticas y fenotípicas entre estas, aplicando el método de correlación intraclase entre medios hermanos de padre (Becker, 1984).

\section{Efectos fijos}

Se utilizó el mismo modelo aditivo lineal en todos los caracteres para analizar la incidencia de los efectos fijos identificados y los respectivos componentes de variancias y covariancias (Modelo I): $Y_{i j k l m}=\mu+T_{i}+S_{j}$ $+A_{k}+E_{l}+e_{i j k l m}$; donde $Y_{i j k l m}^{i j k l m}=$ Valor observado en la m-ésima cría, nacida en la l-ésima estación y el k-ésimo año, del j-ésimo sexo, nacida bajo el i-ésimo tipo de parto; $\mu=\mathrm{Me}-$ dia general de cada característica; $T_{i}=$ Efecto del i-ésimo tipo de parto de la madre; $S_{j}=$ Efecto del j-ésimo sexo de la progenie; $A_{k}=$ Efecto del k-ésimo año de nacimiento de la progenie; $E_{l}=$ Efecto del l-ésimo estación del año de nacimiento; $e_{i j k l m}=$ Efecto aleatorio del error asociado a cada observación.

Se utilizó el procedimiento PROC GLM del Programa SAS v. 8.2 (SAS Institute, 2000), para aislar y dimensionar la incidencia significativa de los efectos fijos considerados sobre los respectivos caracteres en estudio.

\section{Efectos al azar}

Los componentes de varianza y covarianza fueron estimados utilizando el procedimiento PROC VARCOMP del programa SAS v. 8.2 (SAS Institute, 2000), bajo la opción del método de Máxima Verosimilitud Restringida (REML), cuyo modelo operacional mixto (Modelo II) consideró como facto- res fijos al sexo, tipo de parto y la interacción año y estación, y como aleatorios al genotipo de los padres y el error aleatorio: $Y_{i j k l m n}=\mu+$ $T_{i}+S_{j}+A_{k}+E_{l}+\left(A^{*} E\right)_{k l}+P_{m}+e_{i j k l m n}$; donde: $Y_{i j k l m n}=$ Valor del carácter observado en la n-ésima cría, nacida del n-ésimo padre, en la l-ésima estación y el k-ésimo año, del jésimo sexo, nacida bajo el i-ésimo tipo de parto; $\mu=$ Media general de cada característica; $T_{i}=$ Efecto del i-ésimo tipo de parto de la madre; $S_{j}=$ Efecto del j-ésimo sexo de la progenie; $A_{k}=$ Efecto del k-ésimo año de nacimiento de la progenie; $E_{l}=$ Efecto del lésimo estación del año de nacimiento; $\left(A^{*} E\right)_{k l}$ $=$ Efecto de la doble interacción año y estación; $P_{m}=$ Efecto del m-ésimo genotipo del padre; $e_{i j k l m n}=$ Efecto aleatorio del error asociado a cada observación.

\section{Cálculo de los Índices de Selección}

Para la elaboración de índices de selección para corderos y madres, se utilizaron los componentes de variancias y covarianzas genéticas y fenotípicas estimadas para el cálculo de las heredabilidades y correlaciones.

\section{Índices de corderos}

Se propuso dos índices de selección, uno con dos caracteres $\left(I_{1}\right)$ y otro con tres $\left(I_{2}\right)$, estimándose en ambos casos sus respectivos $\mathrm{R}^{2}$. Las características - expresadas en kilogramos - incluidas en los índices fueron, para $\mathrm{I}_{1}$ : Ganancia de Peso del Destete a los 5 Meses (GPD5) y Peso de Beneficio a los 5 Meses (PB); y para $\mathrm{I}_{2}$ : Peso al Destete a los 2 Meses (PD), Ganancia de Peso del Destete a los 5 Meses (GPD5) y Peso de Beneficio a los 5 Meses (PB).

Estos índices asumieron las siguientes expresiones: $\mathrm{I}_{1}=\mathrm{b}_{1}$ *PB $+\mathrm{b}_{2}{ }^{*}$ GPD5 e $\mathrm{I}_{2}=$ $\mathrm{b}_{1}{ }^{*} \mathrm{PD}+\mathrm{b}_{2}{ }^{*} \mathrm{~PB}+\mathrm{b}_{3}{ }^{*} \mathrm{GPD} 5 ;$ donde $\mathrm{b}_{\mathrm{i}}=\mathrm{Co}-$ eficientes de regresión o ponderación para cada carácter $(\mathrm{i}=1,2,3)$. 
Cuadro 2. Precios por unidad y por kilogramo de peso vivo (p.v.) de corderos Hampshire

\begin{tabular}{lc}
\hline Producto & $\begin{array}{c}\text { Precio } \\
(\mathrm{S} / .)\end{array}$ \\
\hline Cordero destetado (cabeza) & 190.00 \\
$1 \mathrm{~kg}$ p.v. de cordero destetado & 12.70 \\
$1 \mathrm{~kg}$ p.v. de cordero 5 meses & 11.00 \\
Incremento extra de 1 kg p.v. & 11.00 \\
Costo de producción $(\%)$ & $65.0 \%$ \\
\hline
\end{tabular}

Fuente: Balance anual promedio de granja

Cuadro 3. Características poblacionales del establecimiento

\begin{tabular}{ll}
\hline Característica & Valor \\
\hline Tasa reproductiva (r) & 0.73 \\
Número de nacidos (n) & 7.0 \\
Cordero de reemplazo (rep) & $1.0^{1}$ \\
Vida útil borrega (años) & 6.4 \\
Prolificidad & 1.4 \\
Número de partos & 5.0 \\
\hline${ }^{1}$ Cada borrega deja al menos un cordero \\
como su reemplazo, a fin de mantener \\
estable el capital pecuario
\end{tabular}

Cuadro 4. Valores económicos por kilogramo de carne, estimados para los caracteres considerados en la estimación de los índices

\begin{tabular}{lc}
\hline Característica & $\begin{array}{c}\text { Valor económico } \\
\text { real (S/.) }\end{array}$ \\
\hline PD $(\mathrm{kg})$ & 18.26 \\
PB $(\mathrm{kg})$ & 15.82 \\
GPD5 $(\mathrm{kg})$ & 15.82 \\
\hline
\end{tabular}

Los coeficientes de ponderación para cada carácter de los índices para corderos, fueron calculados mediante un sistema de ecuaciones simultáneas que, expresado en términos matriciales, fue resuelto mediante el programa MATLAB v. 5.3, con base en: $b_{i}$ $=\mathrm{P}^{-1}$.G.a; donde; $\mathrm{b}_{\mathrm{i}}=$ Coeficiente de ponderación para la i-ésima característica; $\mathrm{P}^{-1}=\mathrm{In}$ versa de matriz de varianzas y covarianzas fenotípicas entre las características incluidas en el índice; $\mathrm{G}=$ Matriz de varianzas y covarianzas genéticas entre las características incluidas en el objetivo de selección; $\mathrm{a}=$ Vector de valores económicos de las características incluidas en el objetivo de selección.

Para la estimación de los valores económicos $\left(\mathrm{a}_{\mathrm{i}}\right)$, se consideró la rentabilidad neta resultante de la producción de una borrega a lo largo de su vida útil, tomándose como referencia las expresiones dadas por Lanari y Mueller (1996), adaptándolas a las condiciones de crianza:

$$
\begin{array}{lll}
\mathrm{PD}: & \mathrm{a}_{\mathrm{PD}}=\mathrm{P}_{\mathrm{PD}} *(\mathrm{r} * \mathrm{n}-\mathrm{rep}) *(1-\mathrm{c}) \\
\mathrm{PB}: & \mathrm{a}_{\mathrm{PB}}=\mathrm{P}_{\mathrm{PB}} *\left(\mathrm{r}^{*} \mathrm{n}-\mathrm{rep}\right) *(1-\mathrm{c}) \\
\text { GPD5: } & \mathrm{a}_{\mathrm{GPD} 5}=\mathrm{P}_{\mathrm{GPD} 5} *(\mathrm{r} * \mathrm{n}-\mathrm{rep}) *(1-\mathrm{c})
\end{array}
$$

donde: $\mathrm{P}_{\mathrm{PD}}=$ Precio de $1 \mathrm{~kg}$ de peso vivo de cordero destetado; $\mathrm{P}_{\mathrm{PB}}=$ Precio de $1 \mathrm{~kg}$ de peso vivo de cordero de 5 meses (carnerillo); $\mathrm{P}_{\mathrm{GPD} 5}=$ Precio estimado del aumento de 1 $\mathrm{kg}$ de peso vivo/animal; $\mathrm{r}=$ Tasa reproductiva; $\mathrm{n}=$ Número de partos por borrega; rep = Número de corderos por borrega que quedan como reemplazo; $\mathrm{c}=$ Costo relativo de producción.

Los cuadros 2, 3 y 4 muestran la estructura de precios de venta y los costos de los productos ovinos, las características poblacionales y los valores económicos reales, estimados para cada uno de los caracteres definidos en los índices de selección propuestos. 
Cuadro 5. Estimados ${ }^{1}$ de correlaciones genéticas y genotípicas entre tamaño de camada al nacimiento (TCN), peso de camada al destete (PCD), peso al destete a los dos meses (PD), peso al beneficio (PB) y ganancia de peso de destete a los cinco meses (GDP5), según 142 registros de ovinos Hampshire de una empresa comercial en Lima, Perú (2002-2004)

\begin{tabular}{cccccc}
\hline Características & TCN & PCD & PD & PB & GPD5 \\
\hline TCN & $0.26 \pm 0.28$ & $0.48 \pm 0.16$ & & & \\
PCD & $0.24 \pm 0.39$ & $0.02 \pm 0.31$ & & & \\
PD & & & $0.60 \pm 0.25$ & $0.78 \pm 0.19$ & $0.40 \pm 0.27$ \\
PB & & & $0.54 \pm 0.13$ & $0.36 \pm 0.21$ & $0.89 \pm 0.32$ \\
GPD5 & & & $0.32 \pm 0.32$ & $0.36 \pm 0.30$ & $0.12 \pm 0.27$ \\
\hline
\end{tabular}

${ }^{1}$ Valores de heredabilidad en la diagonal y correlaciones genéticas y fenotípicas por debajo y por encima de la misma

Cuadro 6. Índices de selección estimados para corderos y borregas Hampshire

\begin{tabular}{clc}
\hline Categorías & Índices de Selección & $\mathrm{r}_{\mathrm{HI}}$ \\
\hline \multirow{2}{*}{ Corderos } & $\mathrm{I}_{1}=4.782 * \mathrm{~PB}+2.864 * \mathrm{GPD} 5$ & 0.415 \\
& $\mathrm{I}_{2}=8.263 * \mathrm{PD}+3.182 * \mathrm{~PB}+4.164 * \mathrm{GPD} 5$ & 0.527 \\
Borregas $^{1}$ & $\mathrm{I}_{3}=\overline{\mathrm{X}}_{\mathrm{r}}+\frac{\mathrm{n} * 0.0153}{1+(\mathrm{n}-1) * 0.0153}\left(\overline{\mathrm{X}}_{\mathrm{j}}-\overline{\mathrm{X}}_{\mathrm{r}}\right\}$ & \\
\hline
\end{tabular}

${ }^{1}$ Asociado al valor genotípico, en función al número de pariciones

\section{Índice de borregas}

Se utilizó un índice de selección masal para la valoración genotípica de borregas (productividad) según el peso de sus camadas al momento del destete. Para ello, se consideró el número de registros (n) y la repetibilidad del carácter $(\mathrm{R})$ :

$$
\mathrm{I}_{\mathrm{i}}=\overline{\mathrm{X}}_{\mathrm{r}}+\frac{\mathrm{n} * \mathrm{R}}{1+(\mathrm{n}-1) * \mathrm{R}}\left(\overline{\mathrm{X}}_{\mathrm{i}}-\overline{\mathrm{X}}_{\mathrm{r}}\right)
$$

donde: $\mathrm{I}$ = Índice de la i-ésima borrega evaluada; $\overline{\mathrm{X}}_{\mathrm{i}}=$ Media móvil del peso de camada al destete nor borrega, para un intervalo de 8 meses; $\overline{\mathrm{X}}_{\mathrm{r}}=$ Promedio de peso de camada al destete del rebaño; $\mathrm{R}=$ Repetibilidad.
La repetibilidad ( $\mathrm{R})$ del peso de camada al destete fue estimada mediante la correlación de medidas repetidas, aislando los componentes de variancia entre borregas $\left(\tau^{2}-\tau^{2} \perp \sigma_{\mathrm{pP}}^{2}\right)$ y del ambiente eventual $\left(\sigma_{\mathrm{e}}^{2}=\sigma_{\mathrm{BE}}^{2}\right)$, obtenidos a través de un análisis de variancia y dividiendo estos, según el procedimiento planteado por Becker (1984); aplicando el procedimiento PROC VARCOMP de SAS (SAS Institute, 2000) para el modelo $Y_{i j}=\mu+I_{i}+e_{i j}$; donde: $Y_{i j}=$ Peso total de camada de la i-ésima borrega en su j-ésimo parto; $\mu=$ Media general del peso total de camada en la población; $I_{i}=$ Efecto de la i-ésima borrega $\left(\sigma_{i}^{2}\right) ; e_{i j}=$ Efecto aleatorio del error asociado a cada observación $\left(\sigma_{e}^{2}\right)$. 


\section{Resultados y Discusión}

\section{Parámetros Genéticos}

Las heredabilidades, correlaciones fenotípicas y genéticas, con sus respectivos errores estándares, se presentan en el Cuadro 5.

\section{Heredabilidad}

Los estimados para TCN y PCD fueron de $0.26 \pm 0.28$ y $0.02 \pm 0.31$, respectivamente; siendo el primero muy superior a los valores de $0.09,0.09-0.11$ y 0.04 , reportados para la especie por Notter (1998), Rao y Notter (2000) y Puntas (2011), respectivamente. El valor de PCD estaba dentro del rango de 0.00 a 0.29 citado para ovinos por Snowder (2007).

Los valores de heredabilidad para características reproductivas de este tipo son de baja magnitud en diversas especies (Falconer, 1981), donde el pequeño tamaño de la población estudiada contribuyó aún más a los valores encontrados, y esto se nota por la magnitud de los errores estándares. Es difícil encontrar poblaciones con mayor número de animales y los pocos establecimientos dedicados a este tipo de crianza no cuentan con registros detallados de datos.

Los valores de $0.60 \pm 0.253$ para PD y $0.12 \pm 0.27$ para GPD5 difieren de los reportados por Notter (1998) en ovinos Suffolk (0.15 y 0.21 , respectivamente). Particularmente, la heredabilidad de PD supera el rango de 0.18 a 0.40 para las razas Suffolk, Dorset Horn, Hampshire y Shropshire Down (Blackwell y Henderson, 1955). En general, las heredabilidades relacionadas con las características productivas encontradas en el presente estudio se encuentran fuera de los rangos señalados en la literatura, a excepción de $\mathrm{PB}$, y podría quedar explicado por la diferencia de los sistemas de producción, número de observaciones y métodos utilizados en su estimación.

\section{Correlaciones genéticas y fenotípicas}

Las correlaciones fenotípicas entre las variables en estudio fueron positivas y con magnitudes de media a alta; asimismo, las correlaciones genéticas también fueron positivas pero de baja a mediana magnitud. Al igual que para el caso de los índices de herencia, no se dispone en el país de este tipo de estimaciones entre características de importancia económica en ovinos Hampshire.

Las correlaciones genéticas y fenotípicas de PD-PB fueron similares a las reportadas por Notter (1998) para la asociación entre peso al destete (60 días) y peso a los tres meses en ovinos Suffolk. Asimismo, este autor obtuvo una alta correlación genética (0.68) entre la ganancia de peso del destete (60 días) y el peso a los 120 días de edad, pero la correlación fenotípica fue negativa (-0.06), probablemente más por efecto materno que por la propia habilidad del cordero de ganar peso. Este mismo comportamiento se observa en el presente estudio, aunque ambas correlaciones fueron positivas (0.32 y 0.40 , respectivamente).

Estos resultados evidenciarían que la selección por mayores pesos al destete conllevaría a mayores pesos vivos a edades posteriores, tanto en estos animales como en su descendencia, como respuesta esperada de la selección; sin embargo, no sería confiable como referente genético o fenotípico de las ganancias de peso esperadas hasta la edad de beneficio, dados los altos errores estándares encontrados en este y otros estudios (Notter, 1998). Además, deben considerarse las diferencias entre poblaciones, su estructura y el cambio que pudiera producirse en el tiempo por efecto de ligamiento (Cardellino y Rovira, 1987).

\section{Repetibilidad}

La repetibilidad del peso de camada al destete (PCD) arrojó un valor de 0.0153 , que es inferior al rango de 0.06 y 0.15 reportados 
para la especie (Blackwell y Henderson, 1955; Fogarty et al., 1985) en diversas razas de ovinos bajo condiciones de pastoreo.

\section{Índices de Selección}

El Cuadro 6 presenta los índices de selección elaborados para corderos $\left(\mathrm{I}_{1}\right.$ e $\left.\mathrm{I}_{2}\right) \mathrm{y}$ borregas $\left(\mathrm{I}_{3}\right)$, así como los coeficientes de correlación con el valor genético verdadero esperado $\left(\mathrm{r}_{\mathrm{HI}}\right)$, derivados de las características consideradas en cada uno de ellos. En el caso de los corderos, la finalidad de los índices se orienta a la identificación de los animales de más alto valor genético con el objetivo de ser reservados como padres de la siguiente generación; y en las borregas, para mantener las de mayor valor genotípico por su capacidad reproductiva en futuras pariciones.

\section{Corderos}

Los coeficientes de regresión para los caracteres PB y GPD5 incluidos en $\mathrm{I}_{1}$ fueron positivos, siendo ligeramente mayor el correspondiente a GPD5 en relación a PB; indicativo de que los corderos con mejores incrementos de pesos tendrían mayor opción de lograr un mayor valor de índice. De igual manera, los coeficientes de regresión de los caracteres PD, PB y GPD5 incluidos en $\mathrm{I}_{2}$ arrojaron valores positivos, siendo $\mathrm{PD}$ la característica de mayor relevancia. En consecuencia, $\mathrm{I}_{2}$ otorga mayor preferencia a los corderos que logran mayores pesos al destete e incrementos de peso del destete a los cinco meses, edad en que se efectúa el beneficio, en relación a aquellos que solamente logran mayores pesos al beneficio. Por lo tanto, se esperaría que la selección derivada de la aplicación de cualquiera de ambos índices generase una tasa de progreso genético positiva para las características involucradas, favoreciendo la selección multicarácter.
Respecto a la eficiencia en el uso de los índices en relación al objetivo de selección, $\mathrm{I}_{1}$ obtuvo un coeficiente de correlación $\mathrm{r}_{\mathrm{HI}}$ inferior al de $\mathrm{I}_{2}$ (Cuadro 6); indicando que la respuesta a la selección en términos de valor genético de los animales se incrementa en un $37.6 \%$ cuando se incorpora el peso al destete en el índice de selección. Sin embargo, debe tenerse en cuenta que los estimados de la heredabilidad y de las correlaciones genéticas y fenotípicas que involucran esta variable pueden estar influenciados por efectos maternos, aun cuando el modelo aplicado consideró el número de parto de las borregas madres. Asimismo, debe considerarse que los coeficientes de regresión hallados en ambos índices responden también a los valores económicos relativos que tienen las características al momento de la venta de los corderos empleados en su estimación.

\section{Borregas}

El índice para borregas $\left(\mathrm{I}_{3}\right)$ considera el PCD como único carácter a seleccionar. Su precisión en la estimación del valor genotípico para producir futuras camadas de mayor peso al destete es función directa de la repetibilidad del carácter y del número de medidas repetidas involucradas (destetes) que la borrega haya logrado en su vida reproductiva. Este hecho le otorga flexibilidad para que el índice sea aplicado prácticamente a cualquier borrega que haya expresado el carácter, indistintamente del número de parto que registre.

La selección de borregas por PCD permite mejorar las características de habilidad materna y prolificidad, y al estar involucrado el peso individual de los corderos en su expresión, es una característica compuesta de mucha utilidad para avanzar de manera balanceada en un plan de mejoramiento genético (Snowder, 2007). El tamaño de camada al nacimiento y el peso de los corderos al destete guardan un fuerte componente materno (Falconer, 1981; Cardellino y Rovira, 1987). 


\section{Conclusiones}

- En general, los parámetros genéticos y fenotípicos estimados se encuentran dentro de los rangos reportados en la literatura, estos últimos más orientados a razas de lana y doble propósito, criados principalmente en praderas bajo condiciones extensivas.

- Los índices derivados se consideran de utilidad para la selección genética de corderos machos y hembras a los cinco meses de edad y para la selección genotípica de borregas en etapa reproductiva.

- La cantidad de información usada no fue suficiente para lograr errores estándares pequeños en los parámetros genéticos y fenotípicos estimados. Sin embargo, los índices genéticos encontrados pueden ser aplicados siempre y cuando no se registren cambios importantes en los precios y en la tendencia del mercado de la carne.

\section{Literatura Citada}

1. Aliaga J. 2006. Producción de ovinos. Lima: Ed Gutenberg. 420 p.

2. Becker AW. 1984. Manual of quantitative genetics. $4^{\text {th }}$ ed. Washington, USA: Academic Enterprises. 190 p.

3. Bianchi A, Garibotto G, Bentancur $O$. 2001. Evaluación de la sobrevivencia, características de crecimiento, peso de la canal y punto GR en corderos pesados Corriedale puros y cruza Texel, Hampshire Down, Southdown y Suffolk. Arch Med Vet 33: 261-268. doi: 10.4067/ S0301-732X2001000200016

4. Blackwell RL, Henderson CR. 1955. Variation in fleece weight, weaning weights and birth weight of sheep under farm conditions. J Anim Sci 14: 831-843.

5. Cardellino R, Rovira J. 1987. Mejoramiento genético animal. Montevideo: Ed Hemisferio Sur. 253 p.

6. Castillo AG. 2003. Evaluación técnica y económica del engorde intensivo de ovinos criollos cruzados con Hampshire Down provenientes de la Sierra. Tesis de Ing. Zootecnista. Lima: Univ Nacional Agraria La Molina. 55 p.

7. Crispim BA, Grisolia AB, Seno LO, Egito AA, Vargas Junior FM, Souza MR. 2013. Genetic diversity of locally adapted sheep from Pantanal region of Mato Grosso do Sul. Genet Mol Res 12: 5458-5466. doi: 10.4238/2013. November.11.7

8. Dickerson GE, Glimp HA, Tuma HJ, Gregory KE. 1972. Genetic resources for efficient meat production in sheep. Growth and carcass characteristics of ram lambs of seven breeds. J Anim Sci 34:940-951.

9. Falconer DS. 1981. Introduction to quantitative genetics. $2^{\text {nd }} e d$. London: Logman. $340 \mathrm{p}$.

10. Fogarty NM, Dickerson GE, Young LD. 1985. Lamb production and its components in pure breeds and composite lines. III. Genetic parameters. J Anim Sci 60: 40-57.

11. Fraser A, Stamp J. 1989. Ganado ovino. Producción y enfermedades. Madrid: Ed Mundi-Prensa. 358 p.

12. García JC. 2013. Perspectiva ovina. [Internet], [14 mayo 2014]. Disponible en: http://www.minagri.gob.cl/wpcontent/uploads/2013/11/Perspectivaovina.pdf

13. [INEI] Instituto Nacional de Estadística e Informática. 2013. IV Censo Nacional Agropecuario 2012: Resultados definitivos. Lima: INEI. $62 \mathrm{p}$.

14. James JW. 1987. Methods of estimating genetic change. In: Proc National Symposium Merino Improvement Programs in Australia. Leura, NSW, Australia. p 47-156.

15. Lanari MR, Mueller JP. 1996. Objetivos de mejoramiento e índices de selección Provino para Corriedale. En: Comunicación Técnica en Producción Animal $N^{\circ}$ 295. San Carlos de Bariloche, Argentina: EEA Bariloche-INTA. p 9-14.

16. MATLAB. 1999. The language of technical computing. Math Works Inc. 
Natick, Massachusetts, USA. [Internet]. Disponible en: http://www.mathworks.com/products/matlab/

17. Mueller J. 1996. Objetivos de mejoramiento de rumiantes menores. En: XXIX Reunión Anual Sociedad Genética de Chile. Viña del Mar.

18. Mueller J. 2001. Mejoramiento genético de las majadas patagónicas. En: Ganadería sustentable en la Patagonia Austral. Borrelli P, Oliva G (eds). Ed INTA Reg Pat Sur. p 209-222.

19. Notter DR. 1998. The US National Sheep Improvement Program: acrossflock genetic evaluations and new trait development. J Anim Sci 76: 2324-2330.

20. [OSU] Oklahoma State University. 2014. Breeds of livestock: sheep. [Internet]. Disponible en: http:// www.ansi.okstate.edu/breeds/sheep/

21. Puntas JA. 2011. Estimación de parámetros genéticos de los criterios de selección del ovino Segureño. [Internet]. Disponible en: http://www.uco.es/orga- niza/departamentos/prod-animal/ economia/aula/img/pictorex/ 28_14_39_Pepe_Puntas.pdf

22. Rao S, Notter DR. 2000. Genetic analysis of litter size in Targhee, Suffolk, and Polypay sheep. J Anim Sci 78: 21132120.

23. Salgado C. 2012. Situación y perspectivas de los mercados de lana y carne ovina. [Internet]. Disponible en: http:// e $\mathrm{x} \mathrm{p}$ o p r a d o . c o m / e s / PDF_ExpoMelilla2012/SUL\%20$\% 20$ Situaci\%F3n\%20y\%20Perspectivas\% $20 \mathrm{de} \% 20 \mathrm{los} \% 20 \mathrm{Mercados} \% 20 \mathrm{de} \%$ 20Lana\%20y\%20Carne\%20Ovina.pdf

24. SAS Institute. 2000. The SAS System for Windows, User's Guide. Statistic, Version 7.12. Cary, NC. USA: Ed SAS Inst.

25. Snowder GD. 2007. Genetic improvement of overall reproductive success in sheep: a review. Arch Latinoam Prod Anim 16: 32-40. 